\title{
HOJAS DE CHAN (Hyptis suaveolens) PARA EL CONTROL DE Sitophilus zeamais Y Zabrotes subfasciatus ${ }^{1}$
}

\author{
Modesto Armando Gómez-Peralta², Jennifer Imelda Lacayo-Morales², Martha Cecilia Rosales-Rivera ${ }^{2}$
}

\section{RESUMEN}

Hojas de chan (Hyptis suaveolens) para el control de Sitophilus zeamais y Zabrotes subfasciatus. Con el objetivo de evaluar el efecto de la hoja de chan sobre Sitophilus zeamais y Zabrotes subfasciatus en granos almacenados de maíz y frijol respectivamente, se estableció un ensayo en condiciones controladas en el año 2007, entre los meses de mayo a setiembre, en la ciudad de León, Nicaragua, a 129 msnm y una temperatura promedio de $29^{\circ} \mathrm{C}$. Se emplearon semillas de frijol de la variedad DOR 364 y semillas de maíz de la variedad NB -6 . Las hojas de chan del estrato medio de las plantas hacia el estrato superior y de tamaño similar, se colectaron antes de la floración se secaron por tres días en un secador solar y luego fueron pulverizadas. Las unidades experimentales consistieron de frascos de plástico de 172,2 g de capacidad, y dimensiones de 7,5 $\mathrm{cm}$ de diámetro por 8 $\mathrm{cm}$ de alto, a los que se adicionaron $100 \mathrm{~g}$ de maíz o $100 \mathrm{~g}$ de frijol. Los tratamientos consistieron en la adición del polvo de hoja seca de chan entre las semillas en seis dosis: 0, 5, 10, 15,20 y 25 gramos por 100 de semilla. Luego se adicionaron 20 adultos seleccionados al azar y sin sexar. Las variables medidas en frijol y maíz fueron: número de insectos muertos; número de granos picados; número de adultos emergidos de los granos y peso del los granos. El polvo de las hojas de chan sólo tuvo efecto en la mortalidad de $S$. zeamays.

Palabras clave: Gorgojos, frijol, maíz, fitoinsecticida,

\section{ABSTRACT}

Leaves of chan (Hyptis suaveloens) for the control of Sitophilus zeamais and Zabrotes subfasciatus. The objective of this work was to evaluate the effect of chan leaves on the survival of Sitophilus zeamais in maize and Zabrotes subfasciatus in bean seeds, during year 2007, between the months of May through September in the city of León, Nicaragua, at 129 masl and an average temperature of 29 ${ }^{\circ} \mathrm{C}$. Bean seeds of the DOR 364 variety and maize seeds NB- 6 were used. Chan leaves of similar size were taken from the middle of the plants toward the top tier, collected before flowering, dried for three days in a solar dryer and then pulverized. The experimental units consisted of plastic bottles of $172.2 \mathrm{~g}$ capacity and dimensions of $7.5 \mathrm{~cm}$ in diameter and $8 \mathrm{~cm}$ high, to which $100 \mathrm{~g}$ of corn or $100 \mathrm{~g}$ of beans were added. The treatments consisted of the addition of dried leaf chan powder in six doses: 0, 5, 10, 15, 20 and 25 grams per 100 seeds. Twenty adult beetles selected randomly and without sexing were added to the jars. The variables measured in beans and corns were: number of dead insects, number of grains perforated, number of adults emerged from the kernels and grains weight. The powdered leaves of chan only had effect on the mortality of $S$. zeamays.

Key words: Beetles, bean, corn, plant bio-insecticide, grain pests. plagas de granos.

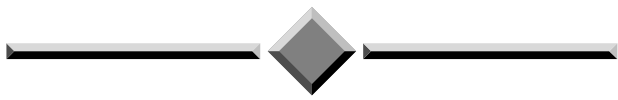

\footnotetext{
Recibido: 1 de agosto, 2009. Aceptado: 16 de noviembre, 2009. Proyecto financiado por RedSICTA, Managua, Nicaragua.

2 Unión Nacional de Agricultores y Ganaderos (UNAG-LEÓN) / Cooperativa. "Los Altos de la Cruz" / grupos "Los Chaneros", León, Nicaragua.modestogmez@yahoo.com.ar; jennifer_lacayo@yahoo.com; marthar81@yahoo.com
} 


\section{INTRODUCCIÓN}

El combate de plagas de granos almacenados con empleo de fosfuro de aluminio es común en Nicaragua a pesar del alto grado de toxicidad para humanos y animales. La exposición de humanos a concentraciones de fosfina en aire de $0,24-2,9 \mathrm{mg} / \mathrm{m}^{3}(0,17-2,11 \mathrm{ppm})$ durante el proceso del tratamiento a granos puede ser letal, o los síntomas pueden perdurar hasta las dos semanas siguientes (Misra et al. 1988). Los productores lo emplean por su efectividad para proteger granos almacenados y fácil aplicación. Pero no pueden comercializar estos granos como orgánicos y dejan de percibir el precio adicional agregado, que pueden variar entre el 10 al $40 \%$ más que los productos bajo la agricultura convencional. Una alternativa al empleo del fosfuro de aluminio está en la repelencia o acción insecticida de extractos de plantas. Las partes de las plantas que se han empleado para la protección de granos almacenado son las hojas, rizomas, tallos, semillas, y frutos, pero en la mayoría de los casos se han usado hojas frescas y secas o en polvo. Hojas frescas o secas son agregados a los granos en proporciones de 1 a $5 \%$ de peso por peso (p/p) y hasta un $18 \%$ para cáscara de cítricos (Don-Pedro 1985, citado por Golob et al. 1999).

El uso de productos de plantas nativas y otros materiales locales disponibles para la protección de cereales y legumbres almacenados, han sido reportados por muchos investigadores (Golob et al. 1982, Stoll 2000, Lale y Abdulrahman 1999). Dentro del rango de especies con propiedades fitoinsecticidas se encuentra el chan (Hyptis suaveolens). Los extractos de hoja fresca de esta planta han sido probados con eficacia para el control de Callosobruchus maculatus. Según Iloba y Ekrakene (2006) usando extractos de hojas de $H$. suaveolens en concentraciones de 1,5 g, 2,5 g y 3,5 g, tuvieron una mortalidad media de $94,3 \%$ cuando usó la concentración más baja; en este caso, $H$. suaveolens fue el mejor para prevenir la emergencia de C. $m a$ culatus y $S$. zeamais comparado con los extractos de Ocimum gratissimum, Azadiracta indica y el control. En el mismo año, Keita et al. (2006), con concentraciones de 1,$25 ; 2,5,5$ y $10 \%$ v/v de la semilla de $H$. suaveolens contra larvas del segundo instar de Plutella xylostella, encontró mortalidades significativas de 63 y $82 \%$ a las 24 y 48 horas respectivamente, en la más alta concentración $(10 \% \mathrm{v} / \mathrm{v})$. El uso del $10 \%(\mathrm{p} / \mathrm{p})$ de polvo de hojas de chan mezclado con semilla de cowpeas redujo el daño del adulto C. maculatus por un período de cuatro meses (Fatope et al. 1995). La repelencia de $H$. suaveolens probablemente se deba a la concentración de aceites mentolados volátiles presentes en las hojas de chan (Perry 1980). Poerzada (1997) reporta un total de 32 componentes secundarios de los cuales 1,8-Cineole (32\%) y Caryophyllene (29\%) son los principales elementos. La toxicidad de 1,8-Cineole hacia tres especies de Coleopteros que atacan granos almacenados fue mostrada por C. maculatus (Aggarwal et al. 2001). La efectividad de $H$. suaveolens puede ser atribuible a éstos $u$ otros metabolitos secundarios los cuales han causado la muerte en gorgojos adultos (Iloba y Ekrakene 2006). También se reporta la eficacia del extracto de hojas de chan para el control de larvas del IV instar de Aedes aegypti (Noegroho et al. 1995). Sobre otros componentes químicos, Golob et al. (1999) menciona que las hojas del género Hyptis poseen un terpenoide llamado limonene con una dosis letal baja en ratas de $4.600 \mathrm{mg}$ (LDL0).

El cultivo del chan es ventajoso para las familias rurales productoras de granos básicos; a la vez que les permite diversificar su producción y obtener hojas para la protección de granos almacenados, pueden obtener alimentos a través de la elaboración de refrescos naturales y para uso medicinal como es el caso del uso de semillas de chan para disminuir o degradar el ácido úrico en la sangre (Aspinall et al. 1991).

El objetivo del presente trabajo fue determinar el efecto de la hoja de chan sobre Sitophilus zeamais en maíz y Zabrotes subfasciatu en frijol.

\section{MATERIALES Y MÉTODOS}

Se ejecutó un proyecto de investigación para evaluar la efectividad del polvo de hoja de chan, en la mortalidad, crecimiento y reproducción de los gorgojos de frijol y maíz Zabrotes subfasciatus y Sitophilus zeamais respectivamente, se efectuó en las instalaciones de la Unión Nacional de Agricultores y Ganaderos (UNAG-León), ubicadas en la ciudad de León, Nicaragua, a $129 \mathrm{msnm}$ y una temperatura promedio de 29 ${ }^{\circ} \mathrm{C}$ entre los meses de mayo a septiembre del año 2007. Se emplearon semillas de frijol de la variedad DOR 364 de color rojo oscuro y granos de maíz de la variedad NB -6 . Las semillas y granos se seleccionaron 
descartando las dañadas o picadas, se limpiaron con una manta y se secaron en un secador solar hasta 14 $\%$ de humedad, luego se almacenaron en recipientes herméticos vidrio con tamaño de $7,5 \mathrm{~cm}$ de diámetro $\mathrm{x}$ $20 \mathrm{~cm}$ de alto a temperatura ambiente durante un mes para descartar la presencia de insectos dentro de las semillas de frijol.

La cría de insectos se inició un mes antes de la aplicación de los tratamientos a las unidades experimentales. Zabrotes subfasciatus fue criado en semillas de la variedad de frijol DOR-364, y Sitophilus zeamais en semillas de maíz de la variedad NB-6. Se emplearon dos tipos de recipiente para la cría de los insectos: un saco de polipropileno con 11 kilos de semilla de maíz o de frijol y ocho vasos de vidrio de $850 \mathrm{~g}$, por cada tipo de semilla (maíz o frijol).

Las hojas de chan del estrato medio de las plantas hacia el estrato superior y de tamaño similar, se colectaron antes de la floración. Las hojas recién cortadas se secaron por tres días en un secador solar (construido con madera y plástico blanco transparente) que alcanzó temperaturas máximas de $60{ }^{\circ} \mathrm{C}$. Luego, los granos de maíz fueron pulverizados con una máquina de moler manualmente, de uso común entre los agricultores.

Las unidades experimentales consistieron de frascos de plástico de 172,2 g de capacidad, a los que se agregaron 100 g de maíz o 100 g de frijol. Se distribuyó el polvo de hoja seca de chan uniformemente entre las semillas y se adicionaron 20 adultos seleccionados al azar y sin sexar. Los frascos se taparon con tela de "manta" (algodón) y sellados con cinta de tejido elástico. Se efectuó una agitación suave de los frascos de vidrio para la circulación de aire dentro de las semillas.

Los tratamientos con polvo de hoja seca de chan, para maíz y frijol fueron los siguientes:

T1: $0 \%$ de polvo de hoja seca por peso del grano $(0 \mathrm{~g})$ $\mathrm{T} 2: 5 \%$ de polvo de hoja seca por peso del grano $(5 \mathrm{~g})$ T3: $10 \%$ de polvo de hoja seca por peso del grano $(10 \mathrm{~g})$

T4: $15 \%$ de polvo de hoja seca por peso del grano $(15 \mathrm{~g})$

T5: $20 \%$ de polvo de hoja seca por peso del grano $(20 \mathrm{~g})$

T6: $25 \%$ de polvo de hoja seca por peso del grano $(25 \mathrm{~g})$

Se empleó un diseño completamente al azar con 10 repeticiones por tratamiento. Las variables medidas en frijol y maíz fueron: 1-número de insectos muertos; 2-número de granos picados; 3-número de adultos emergidos de los granos y 4-peso del los granos.

Los experimentos se dividieron en dos fases para cada especie. En maíz, la Fase I Inició con la ubicación de los insectos en las semillas de maíz. Se realizaron muestreos cada tres días hasta los 15 días después de la introducción de los insectos en las semillas (DDI). En cada muestreo se contó el número de insectos muertos, los cuales se retiraron de los vasos. En el día 15 se realizó el último muestreo de insectos muertos, se cuantificó el número de granos picados y el número de perforaciones por granos. Para estimar el número de perforaciones por grano se tomó una muestra al azar de 20 granos picados, pero cuando el número de granos picados no excedía de los 20 granos se tomo la totalidad de los granos picados para cuantificar el número de perforaciones por grano. Al final de la primera fase se sacaron todos los insectos (vivos y muertos) que aún quedaron en las unidades experimentales. De inmediato se inició la Fase II, cada cinco días se evaluó la emergencia de nuevos adultos hasta 45 días después de iniciada la segunda fase. Al finalizar la segunda fase también se contó el número de granos picados y el número de perforaciones por granos en una muestra al azar de 20 granos picados y se pesaron los granos de cada unidad experimental en una balanza electrónica.

Para estimar la pérdida de peso (Pp) de los granos de maíz se utilizó la fórmula de Adams \& Schulten (1976). Para cuantificar el Pp (\%) se empleo la fórmula: $\mathrm{Pp}=(\mathrm{Ngd} / \mathrm{Ntg}) \times 100 \times \mathrm{C}$. $(\mathrm{Pp}$ : pérdida de peso [\%], Ngd: número de granos dañados, Ntg: número total de granos, C: 0,125 si el maíz es almacenado como grano suelto o mazorca sin brácteas).

En frijol la primera fase se inició con la colocación de 20 adultos de $Z$. subfasciatus en cada unidad experimental. A partir del tercer día de infestación de los granos, se evaluó cada dos días la mortalidad de adultos. Lo insectos muertos se cuantificaron y se retiraron de los frascos. A los 13 días se evaluó el número de granos picados y el número de perforaciones por grano en una muestra al azar de 20 granos picados. La segunda fase se inicio de inmediato para obtener los días a emergencia de nuevos adultos, con base en un conteo cada dos días. Al igual que la primera fase, los insectos emergidos fueron retirados de los frascos hasta que emergió el último insecto. En este momento se cuantificó el número de granos picados y el número 
de perforaciones por grano en una muestra al azar de 20 granos picados. Si el número de granos picados no excedía de los 20 granos, se tomó la totalidad de los granos picados para cuantificar el número de perforaciones por grano.

En maíz en la fase I, la relación número de insectos muertos sobre el número total de insectos por u.e. y la relación número de insectos muertos acumulados sobre el número total de insectos por u.e. fueron procesados con el modelo lineal generalizado bajo el supuesto de distribución binomial (Montgomery 2003, Kachman 2000) se consideró el criterio del análisis de varianza combinado del diseño completamente al azar evaluado periódicamente (Steel et al. 1997, Kuehl 2001), mediante:

$\eta_{i j k}=\log i t\left(\frac{\pi_{i j k}}{1-\pi_{i i k}}\right)=\eta+\alpha_{i}+\tau_{j}+\alpha \tau_{i j}$

i: $1,2 .$. a de muestreo.

$\mathrm{j}: 1,2 . . \mathrm{b}$ de tratamiento.

$\mathrm{k}: 1,2 . . \mathrm{r}$ de réplica.

$\eta_{\mathrm{ijk}}$ : Valor generalizado de una variable de respuesta $\left(Y_{j k}\right)$ medida en el i-ésimo muestreo, el j-ésimo tratamiento y en la k-esima réplica.

$\eta$ : Constante.

$\alpha_{i}$ : Efecto fijo del i-ésimo muestreo.

$\tau_{\mathrm{j}}$ : Efecto fijo del j-ésimo tratamiento.

$\alpha \tau_{\mathrm{ij}}$ : Efecto fijo de la interacción del i-ésimo muestreo y j-ésimo tratamiento.

La relación número de granos picados o perforados sobre el número total de granos evaluados (20 por u.e.) fue procesado con el modelo lineal generalizado bajo el supuesto de distribución binomial y con el criterio de diseño completamente al azar.

$\eta_{j k}=\log i t\left(\frac{\pi_{j k}}{l-\pi_{j k}}\right)=\eta+\tau_{j}$

$\mathrm{j}: 1,2 . . \mathrm{b}$ de tratamiento.

$\mathrm{k}: 1,2 . . \mathrm{R}$ de réplica.

$\eta_{\mathrm{jk}}$ : Valor generalizado de la relación número de granos picados o perforados sobre el número total de granos evaluados $\left(\pi_{\mathrm{jk}}\right)$ del j-ésimo tratamiento y k-esima réplica. $\eta$ : Constante.

$\tau_{\mathrm{j}}$ : Efecto fijo del j-ésimo tratamiento.

El promedio número de perforaciones por grano en maíz fueron procesados con el modelo lineal generalizado bajo el supuesto de distribución de Poisson para la primera variable y gamma para la segunda (Montgomery 2003, Kachman 2000), y con el criterio del diseño completamente al azar:

$\eta_{j k}=\log E\left(Y_{j k}\right)=\eta+\tau_{j}$

$\mathrm{j}: 1,2 \ldots \mathrm{B}$ de tratamiento.

$\mathrm{k}: 1,2 . . \mathrm{r}$ de réplica.

$\eta_{\mathrm{j} k}$ : Valor generalizado de una variable de respuesta $\left(Y_{j k}\right)$ del j-ésimo tratamiento y k-esima réplica.

$\eta$ : Constante.

$\tau_{\mathrm{j}}$ : Efecto fijo del j-ésimo tratamiento.

En la Fase II, el número de insectos emergidos por u.e., y número de insectos emergidos acumulados por u.e., fueron procesados con el modelo lineal generalizado bajo el supuesto de distribución poisson (Montgomery 2003, Kachman 2000) considerando el criterio del análisis de varianza combinado del diseño completamente al azar evaluado periódicamente (Steel et al. 1997, Kuehl 2001).

$$
\eta_{i j k}=\log E\left(Y_{i j k}\right)=\eta+\alpha_{i}+\tau_{j}+\alpha \tau_{i j}
$$

i: 1,2 .. a de muestreo

$\mathrm{j}: 1,2 . . \mathrm{b}$ de tratamiento

$\mathrm{k}: 1,2 . . \mathrm{r}$ de réplica

$\eta_{\mathrm{ijk}}$ : Valor generalizado de una variable de respuesta $\left(Y_{j k}\right)$ medida en el i-ésimo muestreo, el j-ésimo tratamiento y en la k-esima réplica

$\eta$ : Constante

$\alpha_{\mathrm{i}}$ : Efecto fijo del i-ésimo muestreo

$\tau_{\mathrm{j}}$ : Efecto fijo del j-ésimo tratamiento

$\alpha \tau_{\mathrm{ij}}$ : Efecto fijo de la interacción del i-ésimo muestreo y j-ésimo tratamiento

Los granos picados o perforado (\%) por u.e. fue analizado con el modelo lineal generalizado con el supuesto de distribución binomial (Ec. 2), el número de insectos muertos totales por u.e., con el modelo lineal generalizado bajo el supuesto de distribución de poisson y gamma para el número de perforaciones por 
grano y por u.e. (Ec. 3; Montgomery 2003, Kachman 2000). El peso de grano de maíz por u.e. fue procesado con el modelo lineal mixto bajo el supuesto de distribución normal (Steel et al. 1997). El criterio de análisis de las cuatro variables citadas fue el diseño completamente al azar.

La ecuación para el peso de grano fue:

$Y_{j k}=\mu+\tau_{k}+\xi_{j k}$

$\mathrm{j}=1,2, . ., \mathrm{b}$ de tratamiento.

$\mathrm{k}=1,2, . ., \mathrm{r}$ de réplica.

$\mathrm{Y}_{\mathrm{jk}}$ : Valor de una variable de respuesta del j-ésimo tratamiento y k-esima réplica.

$\mu$ : Constante o media común.

$\tau_{\mathrm{k}}:$ Efecto fijo del j-ésimo tratamiento.

$\xi_{\mathrm{jk}}$ : Efecto aleatorio del residuo $\sim \mathrm{DNII}\left(0, \sigma_{r}^{2}\right)$.

Las interacciones de muestreo por tratamiento en variables mortandad de la fase I y II, independientemente de la significación estadística, fue analizada y se obtuvo superficie de respuesta (Cochran y Cox 1992, Montgomery 2003, Steel et al. 1997) mediante la ecuación:

$Y_{i}=\beta_{0}+\beta_{1} X_{i}+\beta_{2} X_{i}^{2}+\beta_{3} X Z_{i}+\beta_{4} Z_{i}+\beta_{5} Z_{i}^{2}+\xi_{i}$

$Y_{i}:$ Valor de la variable mortandad de insectos en la iésima observación.

$X_{i}$ : Valor de variable muestreo (días) en la i-ésima observación.

$Z_{i}$ : Valor de variable tratamiento en la i-ésima observación.

$\beta_{0}, \beta_{1}, \beta_{2}, \beta_{3}, \beta_{4}$ y $\beta_{5}$ : Coeficientes de regresión.

$\xi_{\mathrm{i}}$ : Valor residual en la i-esima observación.

Para obtener las tendencias debido al efecto de los tratamientos se emplearon contrastes ortogonales (en el modelo lineal mixto) y análisis de regresión lineal y cuadrática en variables analizadas con el criterio de diseño completamente al azar. Se empleó el programa SAS v8 (SAS Institute Inc 1999, Pérez 2001).

\section{RESULTADOS Y DISCUSIÓN}

\section{Fase I en maíz}

\section{Número de insectos muertos sobre el número total de insectos}

La relación número de insectos muertos sobre el número total de insectos debido al efecto de hojas de chan, fue significativo $(\mathrm{P} \leq 0,01)$ en sus efectos simples de muestreo (evaluaciones periódicas) y tratamiento. La interacción muestreo por tratamiento, también fue significativa $(\mathrm{P} \leq 0,10)$.

La superficie de respuesta de la mortalidad de Sitophilus zeamais (Figura 1) fue mayor en los primeros diez días de muestreo y se incrementó cuando los tratamientos tuvieron mayor concentración de hojas secas de chan. La mayor mortalidad $(22,13 \%)$ se observó con $25 \%$ de hoja chan y a los cinco días y la menor $(5,70 \%)$ con $0 \%$ de hoja chan y a cinco días.

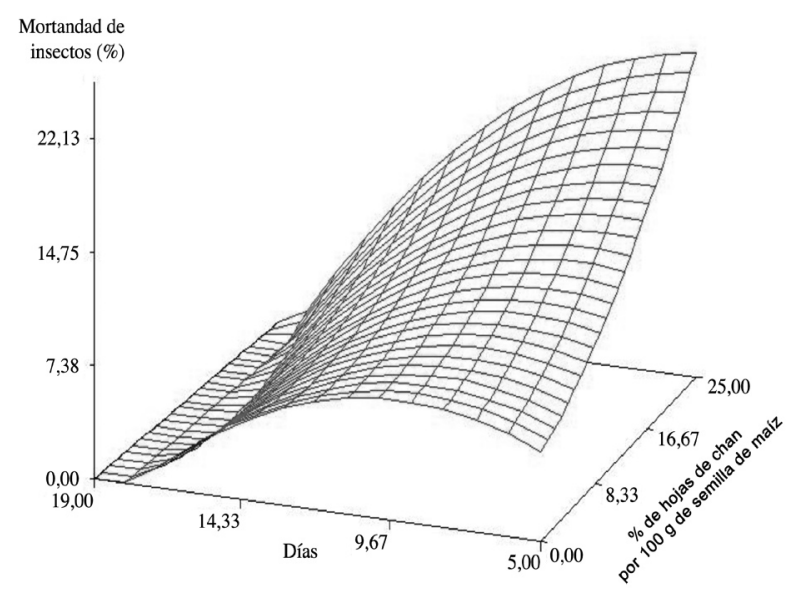

Figura 1. Mortalidad del gorgojo Sitophilus zeamais basado en la relación número de insectos muertos/ número total, por efecto de los tratamientos (hojas secas y molidas de chan Hyptis suaveolens L.Poit) evaluados durante cada tres días durante 15 días. León, Nicaragua 2007. [mortalidad= $-1,718347+(2,030367 *$ días $)+(0,515702 *$ trat $)-$ $\left(0,109149 *\right.$ días $\left.^{2}\right)-(0,045584 *$ trat*días $)$ $+\left(0,014770 * \operatorname{trat}^{2}\right)$ y $\left.\mathrm{R}^{2}: 0,6366\right]$. 
La superficie de respuesta de la mortalidad de insectos presentó una variación explicada de $63,66 \%$ por la variación de los días y las concentraciones de hoja de chan (tratamientos).

\section{Número de insectos muertos acumulados sobre el número total de insectos}

La relación número de insectos muertos acumulados sobre el número total de insectos de gorgojos Sitophilus zeamais por efecto de hojas de chan fue significativa $(\mathrm{P} \leq 0,01)$, por muestreo (evaluaciones periódicas) y tratamiento. La interacción muestreo * tratamiento fue significativa ( $\mathrm{P} \leq 0,05)$, asumiendo efectos independientes de los tratamientos a las evaluaciones periódicas sobre la mortandad de los insectos.

La superficie de respuesta de la mortalidad acumulada de Sitophilus zeamais (Figura 2) fue mayor después de los diez días de muestreo y se incrementó cuando los tratamientos tuvieron mayor concentración de hojas secas de chan. La máxima mortandad $(72,32 \%)$ se observó con $25 \%$ de hoja chan y a 19 días. También a los 19 días con $0 \%$ de hojas chan la mortalidad fue $17,77 \%$. La superficie de respuesta de la mortalidad

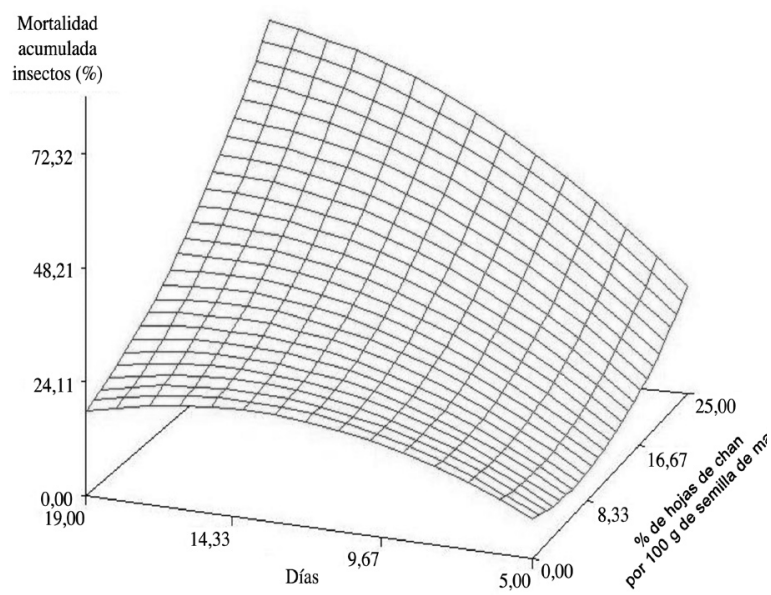

Figura 2. Mortalidad del gorgojo Sitophilus zeamais con base en el número de insectos muertos/número total, durante quince días por efecto de la adición de hojas secas y molidas de chan. León, Nicaragua 2007. [mortalidad $=-12,593047+(5,070385 *$ días $)$ $-(1,246734 *$ trat $)-\left(0,182745 *\right.$ días $\left.^{2}\right)+(0,111596 *$ trat *días $)+\left(0,052333 *\right.$ trat $\left.^{2}\right)$ y $\left.\mathrm{R}^{2}: 0,8976\right]$. de insectos presentó una variación explicada de 89,76 $\%$ por la variación de los días y las concentraciones de hoja de chan (tratamientos).

\section{Relación número de granos perforados sobre el número total de granos evaluados}

La relación número de granos picados o perforados por el gorgojo Sitophilus zeamais sobre el número total de granos evaluados, fue significativa $(\mathrm{P} \leq 0,01)$, con base en las concentraciones de los tratamientos con hoja de chan. Esta respuesta (Figura 3) presentó un incremento hasta el tratamiento de $15 \%$ de chan, para luego disminuir. Así mismo la variación de granos perforados (\%) es explicada en un 72,98 \% por la variación de las concentraciones de los tratamientos.

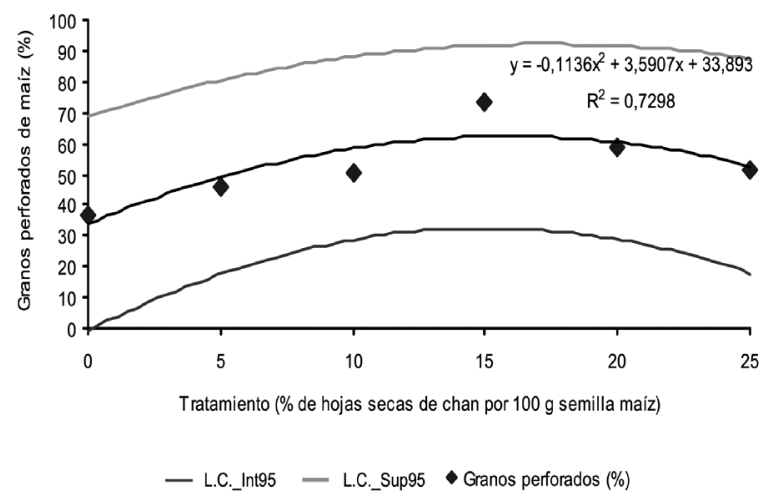

Figura 3. Granos de maíz perforados (\%) por el gorgojo Sitophilus zeamais por efecto de la adición de hojas secas y molidas de chan. León, Nicaragua 2007.

\section{Número de perforaciones por grano de maíz}

El número de perforaciones en granos de maíz, no fue significativo $(\mathrm{P} \leq 0,05)$.

\section{Fase II maíz}

\section{Número de insectos emergidos}

El número de gorgojos emergidos (Sitophilus zeamais) fue significativo $(\mathrm{P} \leq 0,01)$ en sus efectos simples e interacción del muestreo (Evaluaciones periódicas) 
y tratamiento. Para la interacción se deducen efectos dependientes de los tratamientos a las evaluaciones periódicas sobre el número de gorgojos emergidos.

La emergencia de los gorgojos en la segunda fase mayor en el tratamiento con $0 \%$ de concentración de hojas de chan en todas las evaluaciones o muestreos (Figura 4). La emergencia de los gorgojos disminuyó a medida que aumentaron las concentraciones de los tratamientos. La superficie de respuesta de la emergencia de los gorgojos presentó una variación explicada de $48,82 \%$ por la variación de los días y las concentraciones de hoja de chan.

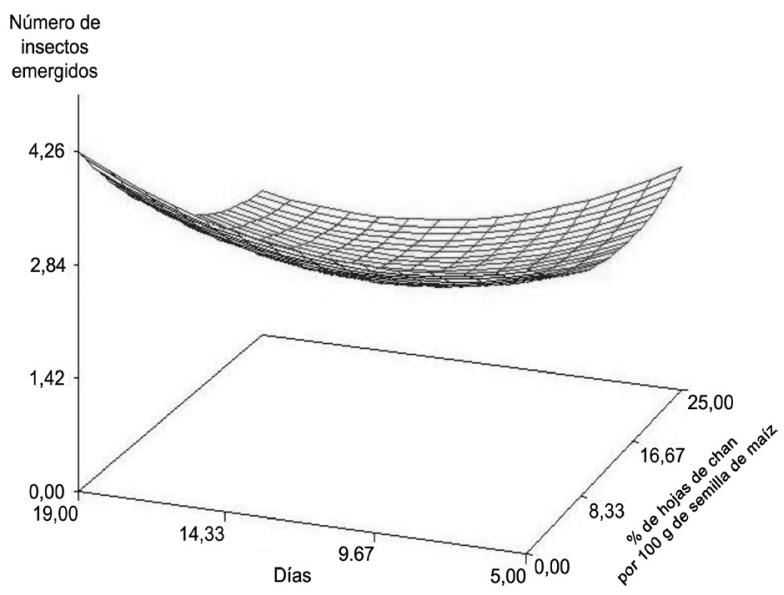

Figura 4. Número de insectos emergidos (Sitophilus zeamais) durante quince días bajo el efecto de la adición de hojas secas y molidas de chan. León, Nicaragua 2007. [insectos $=4,239372$ $(0,207842 *$ días $)-(0,084388 *$ trat $)+(0,010988 *$ día $\left.\mathrm{s}^{2}\right)-(0,004993 *$ trat*días $)+\left(0,003488 *\right.$ trat $\left.^{2}\right)$ y $\mathrm{R}^{2}$ : $0,4882]$.

\section{Número acumulado de insectos emergidos}

El número acumulado de gorgojos emergidos (Sitophilus zeamais), fue significativa $(\mathrm{P} \leq 0,01)$ en sus efectos simples e interacción del muestreo (evaluaciones periódicas) y tratamiento. Con efectos dependientes de los tratamientos y las evaluaciones periódicas sobre el número de gorgojos emergidos.

La emergencia de los gorgojos en la segunda fase fue mayor en el tratamiento con $0 \%$ de concentración de hojas de chan en todas las evaluaciones o muestreos (Figura 5). La emergencia de los gorgojos disminuyó conforme aumento la concentración de chan hasta el $15 \%$. Luego aumentó la emergencia de los gorgojos. La superficie de respuesta de la emergencia de los gorgojos presentó una variación explicada de 91,99\% por la variación de los días y las concentraciones de hoja de chan (tratamientos).

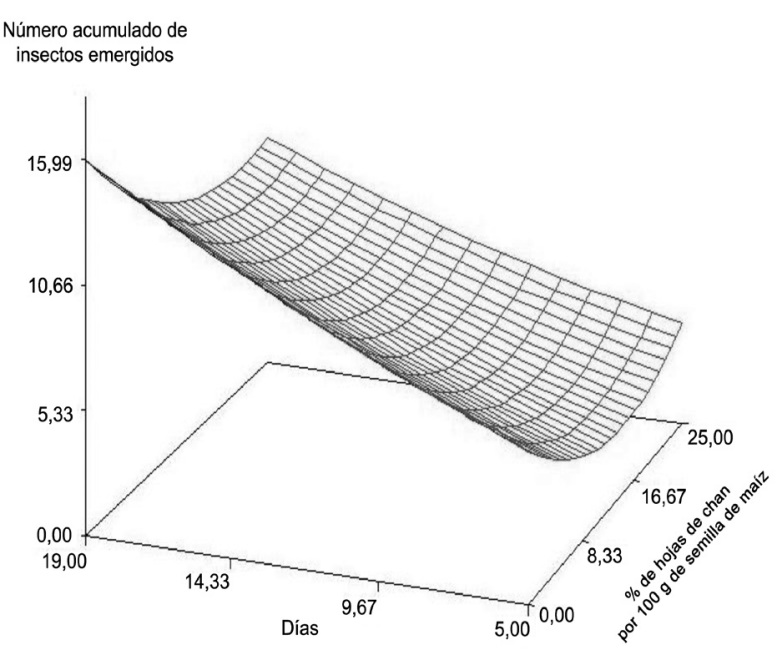

Figura 5. Número acumulado de insectos emergidos (Sitophilus zeamais) durante quince días bajo el efecto de la adición de hojas secas y molidas de chan. León, Nicaragua 2007. [insectos $=3,887150+$ $(0,446789 *$ días $)-(0,410796 *$ trat $)+\left(0,010 *\right.$ días $\left.^{2}\right)$ $-(0,010638 *$ trat*días $)+\left(0,015500 * \operatorname{trat}^{2}\right)$ y $\mathrm{R}^{2}$ : $0,9199]$.

\section{Granos de maíz picados o perforados (\%)}

En el número de granos de maíz perforados hubo efecto significativo $(\mathrm{P} \leq 0,01)$ por efecto de las concentraciones de hoja chan (tratamiento). Conforme se aumentó su concentración se disminuyó en un $0,36 \%$ los granos perforados (Figura 6), por tres unidades de concentración de hoja de chan disminuyó aproximadamente en $1 \%$ el grano perforado. En relación con el coeficiente de determinación, se observó 63,57 \% de variación del grano perforado (\%) es explicada por la variación de las concentraciones de hoja de chan de los tratamientos. 


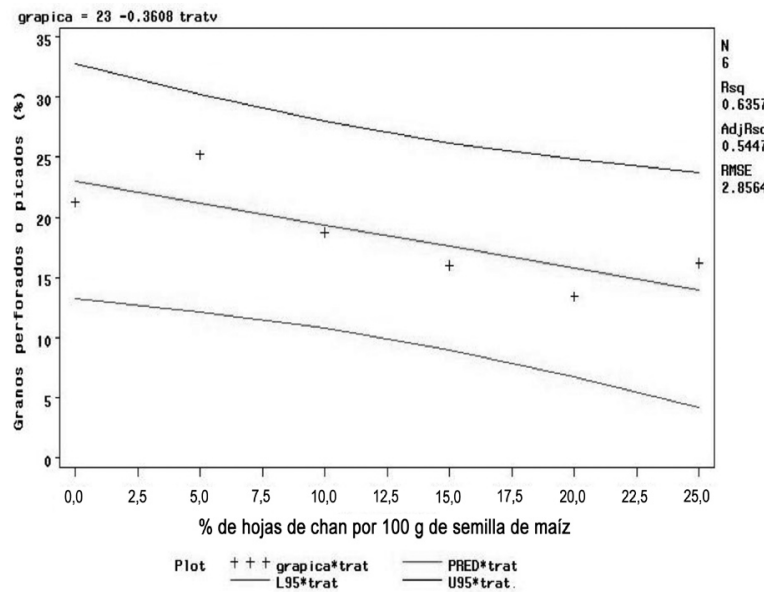

Figura 6. Número de granos de maíz perforados por Sitophilus zeamais bajo el efecto de hojas secas y molidas de chan. León, Nicaragua. 2007.

\section{Número de perforaciones por grano de maíz}

El número de perforaciones por grano de maíz fue significativa $(\mathrm{P} \leq 0,10)$ por efecto de las concentraciones de hoja chan. Por cada aumento disminuyeron en 0,004 perforaciones por grano (Figura 7). Respecto al coeficiente de determinación se observó $26,90 \%$ de variación del número de grano perforado es explicada

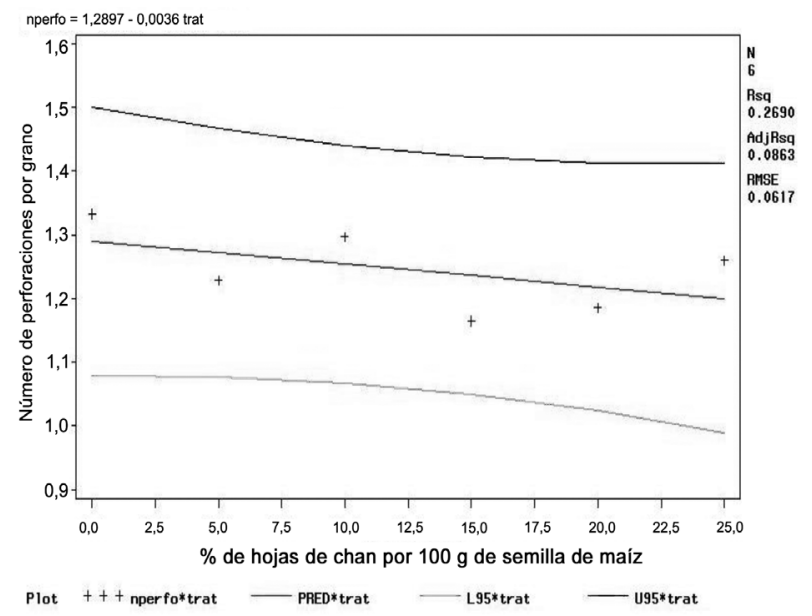

Figura 7. Número de perforaciones en granos de maíz por Sitophilus zeamais bajo el efecto repelente de hojas secas y molidas de chan. León, Nicaragua 2007. por la variación de las concentraciones de hoja de chan de los tratamientos.

\section{Peso de grano de maíz (g)}

El peso del grano de maíz aumento por efecto del aumento en la concentración de hojas secas de chan $(\mathrm{P} \leq 0,10)$ (Figura 8). Por cada $12 \%$ de hoja de éste aplicada se aumentó un gramo aproximadamente, el peso de las semillas de maíz.

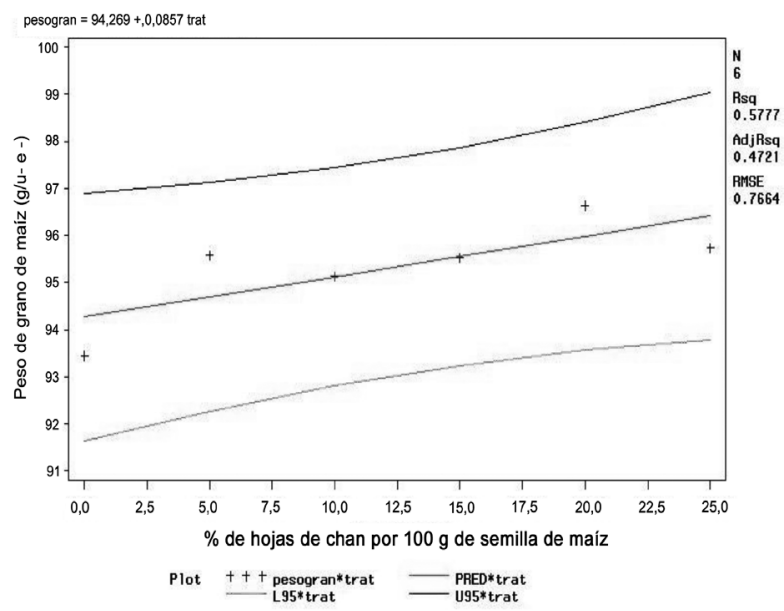

Figura 8. Peso de grano de maíz bajo el efecto de hojas secas y molidas de chan como repelente del gorgojo Sitophilus zeamais. León, Nicaragua 2007.

\section{Fase I, frijol}

Se efectuó análisis de regresión para conocer la naturaleza de la tendencia que siguieron los valores de insectos muertos en dependencia de los porcentajes de hoja de chan utilizados en el experimento, con el fin de determinar el porcentaje de polvo de hoja de chan que es fue más efectivo para el control del gorgojo del frijol.

Para determinar si existió una relación entre los niveles de polvo de chan y los insectos muertos, los granos picados y el número de perforaciones por grano, los datos originales fueron trasformados a Raíz cuadrada de $X$ más 0,5 . Este nuevo conjunto de datos fue sometido a análisis de varianza de la regresión, con el propósito de obtener información sobre el tipo de relación existente entre la variable independiente 
(porcentaje de hojas molidas de chan) y las variables dependientes (insectos muertos, granos picados y perforaciones por grano).

El valor de probabilidad $>$ F (1) obtenida fue de 0,$1515 ; 0,0616$ y 0,3881 para las variables granos picados, perforaciones en el grano e insectos muertos, respectivamente, en los tres casos evaluados la regresión no fue significativa. El R cuadrado fue de 0,035; 0,058 y 0,012 , respectivamente para granos picados, perforaciones en el grano e insectos muertos, lo que indica que no existió ningún efecto de los porcentajes de polvo de hoja de chan utilizados en el experimento para el control de los gorgojos del grano.

En vista que la mortalidad de insectos fue medida en diferentes momentos con base en la misma muestra, se realizo el análisis de medidas repetidas por medio de componentes de varianza, con el propósito de observar diferencias en la mortalidad de los insectos en las diferentes fecha de muestro ( $\mathrm{pr}>0,0001)$. Los resultados del análisis muestran que existieron diferencias significativas en la mortalidad de los insectos en las diferentes etapas de muestro, sin embargo, una vez más el resultado del análisis no mostró diferencias entre los tratamientos evaluados (porcentajes de polvo de hojas de chan). El análisis muestra diferencias en la mortalidad de gorgojos en las diferentes fechas de muestreo, lo cual no puede ser atribuido al efecto de los tratamientos, debido a que la interacción entre tratamientos y la fecha de conteo no fue significativa $(\mathrm{pr}=0,1290)$.

\section{Fase II Frijol}

Las medias de gorgojos emergidos (acumulados en nueve fechas) variaron entre 0,8 y 1,3 insectos emergidos, con cuatro tratamientos con la misma media $(1,3)$. Por tanto, no se evidencia diferencias entre los tratamientos.

$\mathrm{Al}$ igual que para los datos de la fase I, los datos de la fase II fueron sometidos a pruebas estadísticas con el propósito de obtener in formación sobre normalidad y heterogeneidad de varianza. Las salidas de SAS muestran que en ambos casos los datos no son normales y las varianzas heterogéneas, por tanto para proseguir con los análisis se realizo transformación de las variables a raíz cuadrada de X más 0,5 Con el propósito de determinar diferencias entre tratamientos respecto a las variables perforaciones de los granos y granos picados, se realizó una prueba de kruskall
Wallis. En ambos casos hubo diferencias significativas entre tratamientos al $5 \%$ de significancia $(\mathrm{P}=0,0312$ y 0,0446$)$, se observa que los valores obtenidos no definen una tendencia al incrementar el porcentaje de polvo de hojas de chan utilizadas en el experimento.

Se evaluó el grado de dependencia entre los diferentes porcentajes de hoja de chan y las variables granos picados e gorgojos muertos. Ambas pruebas mostraron que no existió evidencia de que la mortalidad de insectos y los granos picados dependiera de los porcentajes de hoja de chan utilizados como tratamientos en el experimento.

El análisis orientado a determinar la diferencia en la mortalidad de los insectos en el tiempo mostro diferencias estadísticas significativas entre las diferentes fechas de evaluación. Sin embargo, este resultado fue irrelevante ya que no evidencia el efecto del porcentaje de polvo de hoja de chan que afecta la población del gorgojo del frijol. La interacción entre tratamientos y tiempo de muestreo fue no significativa, por tanto la mortalidad de gorgojos en las diferentes fechas de muestreo no puede ser atribuida al efecto de los tratamientos.

El análisis de los datos recopilados durante la segunda fase del estudio muestra resultados similares a los arrojados durante la I fase. El resultado del análisis no presentó una clara tendencia del efecto de los tratamientos sobre las variables evaluadas.

\section{CONCLUSIONES}

En este experimento, el uso de polvo de hoja de chan fue efectivo para proteger granos almacenados de maíz al incrementar la mortalidad y disminuir la emergencia de gorgojos de S. zeamais. Pero no fue efectivo contra el gorgojo del frijol Z. subfasciatus.

La efectividad en el uso de hojas de chan para el control de $S$. zeamais se demuestra en el incremento de la mortalidad de adultos cuando se incrementó la concentración de hojas de chan. La máxima mortalidad acumulada (72,32\%) se observó con la concentración de $25 \%$ de hojas chan a los 19 días después de la infestación de los granos.

La emergencia de los gorgojos ( $S$. zeamais) en la segunda fase mayor en el tratamiento $0 \%$ de concentración de hojas. La emergencia de los gorgojos disminuyó a medida que se aumentó la concentración de hojas de chan. 


\section{AGRADECIMIENTOS}

Un agradecimiento especial a los productores de la cooperativa "Los Altos de la Cruz" y al grupo de productores "Los Chaneros" por su participación activa y colaboración en este proyecto; a los técnicos y estudiantes que participaron en la ejecución de las diferentes actividades y fases del proyecto, a la UNAGLeón por su contribución para hacer posible esta iniciativa y finalmente un agradecimiento al apoyo financiero y seguimiento técnico obtenido de parte del proyecto Red SICTA del IICA, así como a Armando Ferrufino, Telma Brenes y Miguel Altamirano, quienes participaron activamente en la planeación del trabajo.

\section{LITERATURA CITADA}

Adams, JM; Schulten, CGM. 1976. Losses caused by insects, mites and microorganismos. In: Postharvest Grain Loss Assement Methods. American Association of Cereal Chemists Sloogh, England. p. 83-93.

Aggarwal, KK, Tripathi, KA; Prajapati, V; Sushil, K. 2001. Toxicity of 1,8 -Cineole towars three species of stored product coleopterans. Insect Science and its Application 21:155-160.

Aspinall, GO; Capek, P; Carpenter, RC; Gowda, DC; Szafranek, J. 1991. A novel L-fuco-4-O-methylD-glucurono-D-xylan from Hyptis suaveolens. In: Carbohydr Res 214(1):107-113.

Cochran,W;Cox,GM. 1992.Estadísticaparala administración y la economía: Diseño de experimentos y análisis de varianza. 10 ed - 2008- 1056 p. Cengage Learneing Editores 2008. p. 490.

Don-Pedro, KN. 1985. Toxicity of some citrus peels to Dermestes maculatus Deg and Callosobruchus maculatus. J. Stored Product Research 21(1):31-34.

Fatope, MO; Nuhu, AM; Mann, A; Takeda, Y. Inter. J. Pest Manag. 1995. Cowpea weevil biossay: A simple prescreen for plants with grain protectant effects. International Journal of Pest Management 41(2):84-86.

Golob, P; Mwanbula, J; Mlango, V; Ngulube, F. 1982. The use of locally available materials as protectants of maize grains against insect infectation during storage un Malawi. Journal of Stored Products Research 18:67-78.

Golob, P; Gudrups, I; Moss, C; Dales, M; Fidgen, A; Evans, J. 1999. The use of spices and medicinals as bioactive protectans for grains. (en línea). FAO. Agricultural services Bulletin No. 137. Rome: Food and Agriculture
Organization. Consultado: 6 oct. 2009. Disponible: http://www.fao.org/docrep/x2230e/x2230e00.htm.

Iloba, BN; Ekrakene, T. 2006. Comparative Assessment of Insecticidal Effect of Azadirachta indica, Hyptis suaveolens and Occimun gratissimun o Soto philus zeamais and Callosobruchus maculates. Journal of Biological Sciences 6(3):626-630.

Kachman, S. 2000. An introduction to generalized linear mixed models. (en línea). Department of Biometry, University of Nebraska-Lincoln. 15 p. Consultado: 12 oct. 2009. Disponible en: http://statistics.unl.edu/ faculty/steve/glmm/paper.pdf

Keita, SS; Umoetok, BA; Smith, JG. 2006. The insecticidal activity of petroleum ether extract of Hyptis usaveolens Poit. (Labiatae) seed on Plutella xylostella (L.) (Lepidoptera: Y ponomeutidae). Agricultural Journal 1(1):11-13.

Kuehl, R. 2001. Diseño de experimentos. Principios estadísticos de diseño y análisis de investigación. Segunda edición. Traducción Marcia González. Thomson Editores, S. A. México. D. F. 666 p.

Lale, NES; Abdulrahman, T. 1999. Evaluation of neem (Azadirachta indica A. Juss) seed oil obtained by different methods and neem powder for the management of Callosobruchus maculatus (F.) (Coleoptera: Bruchidae) in stored cowpea. (En línea). Journal of Stored Products Research 35(2):135-14. Consultado: 12 oct. 2009. Disponible en: http://www.sciencedirect.com/science

Misra UK; Bhargava, SK; Nag, D; Kidwai, MM; Lal, MM. 1988. Occupational phosphine exposure in Indian workers. Toxicology Letters 42(3):257-263.

Montgomery, D. 2003. Diseño y análisis de experimentos. Trad. Rodolfo Piña García. Limusa Wiley México D.F. 686 p.

Neogrobo, SP; Srimulyani dan Mulyaningsih, B. 1995. Larvaside activity of jukut essensial oil Hyptis suaveolens (L) poit leaves toward instar IV of Aedes aegypti larvae and gas chromatography-mass spectroscopy analysis.(en Línea). Indonesian Journal of Pharmacy Majalah Farmasi Indonesia (MFI) 8(4). Artículo 3., 11 p. Consultado: 12 nov. 2009. Disponible en: http://ugm2.tripod.com/mfi84.htm

Pérez, C. 2001. El sistema estadístico SAS®. Editorial Pearson Educación S.A. Prentice Hall. Madrid. p. 88.

Perry, LM. 1980. Medicinal plants of East and Southeast. Asia. (En línea) MIT Press (Cambridge). 620 p. Consultado: 6 nov. 2009. Disponible en: http:// openlibrary.org/b/OL4421389M/Medicinal_plants_ of_East_and_Southeast_Asia 
Poerzada, N. 1997. Chemical composition of the essential oil of Hyptis suaveolens. (en línea). Molecules 2:165168. Sallam, MN. 1999. Chapter 11 Insect Damage on Post-harvest. Consultado: 12 oct. 2009. Disponible: http://www.fao.org/inphol.

SAS Institute Inc. 1999. SAS On lineDoc Versión 8. (En línea). Cary, NC, USA. Consultado: 12 nov. 2009.
Disponible en: http://v8doc.sas.com/sashtml/

Steel, R; Torrie, J; Dickey, D. 1997. Principles and procedures of statistics abiometrical approach. 3 ed. The McGraw-Hill Companies, Inc. USA. 666 p.

Stoll, G. 2000. Natural crop protection in the tropics. letting information come to life. 2 ed. Margraf Verlag, Weikersheim,Germany. 376 p. 
